\title{
Reduction of the transverse effective charge of optical phonons in ZnO under pressure
}

Cite as: Appl. Phys. Lett. 96, 231906 (2010); https://doi.org/10.1063/1.3447798

Submitted: 19 April 2010 . Accepted: 18 May 2010. Published Online: 09 June 2010

J. S. Reparaz, L. R. Muniz, M. R. Wagner, A. R. Goñi, M. I. Alonso, A. Hoffmann, and B. K. Meyer

\section{ARTICLES YOU MAY BE INTERESTED IN}

A comprehensive review of $\mathrm{ZnO}$ materials and devices

Journal of Applied Physics 98, 041301 (2005); https://doi.org/10.1063/1.1992666

Phonon deformation potentials in wurtzite GaN and ZnO determined by uniaxial pressure dependent Raman measurements

Applied Physics Letters 98, 061906 (2011); https://doi.org/10.1063/1.3554434

On the optical band gap of zinc oxide

Journal of Applied Physics 83, 5447 (1998); https://doi.org/10.1063/1.367375

Lock-in Amplifiers Find out more today

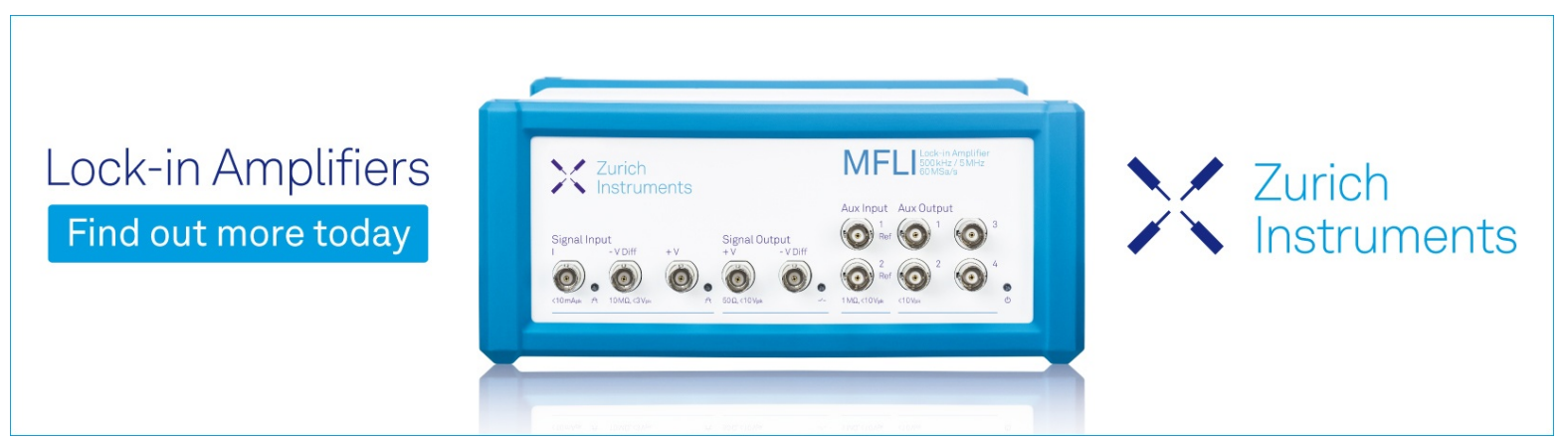




\title{
Reduction of the transverse effective charge of optical phonons in $\mathrm{ZnO}$ under pressure
}

\author{
J. S. Reparaz, ${ }^{1, a)}$ L. R. Muniz, ${ }^{2}$ M. R. Wagner, ${ }^{1}$ A. R. Goñi, ${ }^{2}$ M. I. Alonso, ${ }^{2}$ A. Hoffmann, ${ }^{1}$ \\ and B. K. Meyer ${ }^{3}$ \\ ${ }_{1}^{1}$ Institut für Festkörperphysik, Technische Universität Berlin, Hardenbergstr. 36, 10623 Berlin, Germany \\ ${ }^{2}$ Institut de Ciència de Materials de Barcelona-CSIC, Esfera UAB, 08193 Bellaterra, Spain \\ ${ }^{3}$ I. Physics Institute, Justus Liebig University, Heinrich-Buff-Ring 16, 35592 Giessen, Germany
}

(Received 19 April 2010; accepted 18 May 2010; published online 9 June 2010)

\begin{abstract}
From Raman scattering on a-plane wurtzite $\mathrm{ZnO}$ crystals we obtained a decreasing splitting between longitudinal and transversal optical phonons with $A_{1}$ and $E_{1}$ symmetry as a function of hydrostatic pressure up to $5.5 \mathrm{GPa}$. Consequently, the transverse effective charge $\left(e_{T}^{*}\right)$ exhibits a strong reduction with increasing pressure, yielding $2.17-14.6 \times 10^{-3} \mathrm{P} / \mathrm{GPa}$ and $2.04-13.7$ $\times 10^{-3} \mathrm{P} / \mathrm{GPa}$ (in units of the elementary charge) for the $A_{1}$ and $E_{1}$ phonons, respectively. We find a clear systematic in the linear pressure coefficient of $e_{T}^{*}$ with bond polarity for the series of wide-band gap semiconductors SiC, AlN, GaN, and ZnO. (C) 2010 American Institute of Physics. [doi:10.1063/1.3447798]
\end{abstract}

$\mathrm{ZnO}$ is a semiconductor material which has drawn renewed attention due to its promising integration into optoelectronic devices. Its large band gap of about $3.3 \mathrm{eV}$ at room temperature, its structural compatibility with $\mathrm{GaN}$, and its low production cost are some of the properties that make this system interesting for sensor applications. ${ }^{1}$ In addition, its nontoxicity and biocompatibility with organic systems have been only recently discussed, ${ }^{2,3}$ pointing to $\mathrm{ZnO}$ as a promising candidate for hybrid devices. For many applications a fundamental understanding of the thermal, optoelectronic, and mechanical properties of this system is mandatory. At ambient conditions the thermodynamically stable crystal phase of $\mathrm{ZnO}$ is the wurtzite structure. Recently, the mechanical properties of $\mathrm{ZnO}$ have been investigated up to 60 $\mathrm{GPa}^{4}$ The initial wurtzite phase was observed to transform to the rocksalt structure around 9.1 GPa with a large volume collapse of $16.7 \%$ on increasing pressure. The wurtzite structure belongs to the $C_{6 v}^{4}$ space group with four atoms per unit cell. From the 12 possible vibrational modes only the $A_{1}, E_{1}$, and $E_{2}$ are Raman-active optical modes. The ionic character of the $\mathrm{Zn}-\mathrm{O}$ bonds is responsible for the large splitting of the polar modes $\left(A_{1}\right.$ and $\left.E_{1}\right)$ into longitudinal optical (LO) and transverse optical (TO). The Born's transverse effective charge $\left(e_{T}^{*}\right)$ of the lattice ions is determined by the LO-TO splitting, the screening of the Coulomb interaction (which depends on the electronic part of the dielectric function $\epsilon_{\infty}$ ), and the volume of the unit cell. ${ }^{5}$ All three parameters depend on pressure but is the LO-TO splitting the one dictating the qualitative behavior of $e_{T}^{*}$ at reduced volumes.

In a recent work ${ }^{6}$ the hydrostatic pressure dependence of the Raman optical modes of wurtzite $\mathrm{ZnO}$ was investigated up to $9 \mathrm{GPa}$. Surprisingly, the authors found that the LO-TO splitting increases with increasing pressure for the $E_{1}$ modes. This result is in clear contrast with observations made for other II-VI compounds such as ZnSe (Ref. 7) and ZnTe, where the LO-TO splitting decreases with pressure. The Born's transverse effective charge (which is computed from the value of the LO-TO splitting) was also shown to increase

${ }^{\text {a)} E l e c t r o n i c ~ m a i l: ~ j s r e p a r a z @ g m a i l . c o m . ~}$ with pressure $\left(d e_{T}^{*} / d P>0\right)$. This result is again difficult to match with previous observations in $\mathrm{GaN},{ }^{8} \mathrm{AlN},{ }^{8} \mathrm{GaAs},{ }^{9}$ and $\mathrm{GaP}^{10}$ where $e_{T}^{*}$ was shown to decrease with pressure or at most to remain nearly constant. So far the only known material which exhibits a positive pressure derivative $d e_{T}^{*} / d P$ $>0$ is $\mathrm{SiC}$. In this particular case, this effect was suggested to be a consequence of the lack of $p$ electrons in the carbon cores, which allows for a larger penetration of the Si wave functions into that regions. ${ }^{8,11}$ In spite of the fact that the same argument would apply to the oxygen cores of $\mathrm{ZnO}$, the problem is that the reported value for $d e_{T}^{*} / d P$ in Ref. 6 is larger than that of $\mathrm{SiC}$ even though the bond polarity of $\mathrm{ZnO}$ is much higher than for $\mathrm{SiC}$ and for the nitrides as well, which exhibit $d e_{T}^{*} / d P \approx 0$. This apparent inconsistency was a strong motivation for revisiting the pressure dependence of $e_{T}^{*}$ in $\mathrm{ZnO}$ crystals.

In this letter, we report the dependence on hydrostatic pressure of the zone-center optical phonons of wurtzite $\mathrm{ZnO}$ crystals as obtained from Raman measurements. From a detailed line shape analysis of the Raman spectra we were able to determine the Grüneisen parameters as well as the pressure dependence of the LO-TO splitting for both polar $A_{1}$ and $E_{1}$ modes. The Born's transverse effective charge was found to decrease with increasing pressure for both modes, displaying a clear systematic behavior of $d e_{T}^{*} / d P$ as a function of bond polarity (as defined in Ref. 12) for the whole series of materials from $\mathrm{SiC}, \mathrm{AlN}$, and $\mathrm{GaN}$ to $\mathrm{ZnO}$.

Raman spectra were collected at room temperature with a LabRam HR800 system in backscattering geometry from an a-plane surface. In this configuration the $c$ axis of the $\mathrm{ZnO}$ crystal is perpendicular to the direction of incidence of the laser light. The $514.5 \mathrm{~nm}$ line of an $\mathrm{Ar}^{+}$laser was used for excitation, focused onto the sample using a long distance $20 \times$ Olympus objective. Raman peak positions were determined with an error of less than $0.5 \mathrm{~cm}^{-1}$. Measurements under pressure were carried out using the diamond anvil cell (DAC) technique. A 4:1 mixture of methanol and ethanol was employed as the pressure-transmitting medium. Pressure was monitored in situ by the shift in the ruby $\mathrm{R}_{1}$ line. ${ }^{13} \mathrm{An}$ a-plane wurtzite $\mathrm{ZnO}$ crystal purchased from Crystec was 


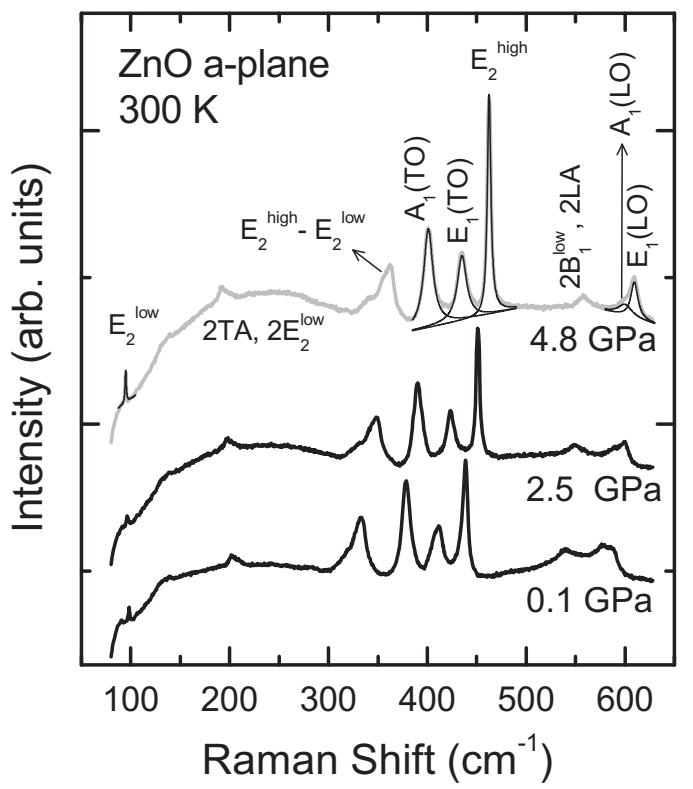

FIG. 1. Representative Raman spectra of an a-plane wurtzite $\mathrm{ZnO}$ crystal at three different pressures of $0.1,2.5$, and $4.8 \mathrm{GPa}$. The spectra were vertically shifted for clarity. Fits to the first order Raman peaks are shown as example for the $4.8 \mathrm{GPa}$ spectrum.

thinned to about $30 \mu m$ by mechanical polishing and loaded into the DAC.

Figure 1 shows three representative Raman spectra from the a-plane surface at different pressures of $0.1,2.5$, and 4.8 $\mathrm{GPa}$. For this configuration the only allowed optical Raman modes are the $E_{2}^{\text {high }}, E_{2}^{\text {low }}, A_{1}(\mathrm{TO})$, and $E_{1}(\mathrm{TO}) .{ }^{14}$ Nevertheless, the $A_{1}(\mathrm{LO})$ and $E_{1}(\mathrm{LO})$ are also observed in the spectra due to the large angular aperture of the focusing objective $(\mathrm{NA}=0.35)$, which leads to a partial lifting of selection rules by departure from strict normal incidence. In the present case, this represents an advantage since it allows for a precise determination of the LO-TO splittings. The peak positions were determined by fitting every spectrum using Lorentzian line shapes for $A_{1}, E_{1}$, and $E_{2}^{\text {low }}$ phonons. For the $E_{2}^{\text {high }}$ mode, however, we used a Fano profile, since in the lower pressure regime $(\mathrm{P}<3 \mathrm{GPa})$ its line shape is strongly influenced by anharmonic effects, due to its decay into a sum of transverse and longitudinal acoustic phonons $(\mathrm{TA}+\mathrm{LA})$ in the vicinity of the $K$ point of the Brillouin zone. ${ }^{15}$

Figure 2 displays the dependence on hydrostatic pressure of the main first-order Raman modes. We only show data in the pressure range up to $5.5 \mathrm{GPa}$, for which the pressure dependence of all peaks is well described by a straight line. The solid lines in Fig. 2 represent the results of least-squares

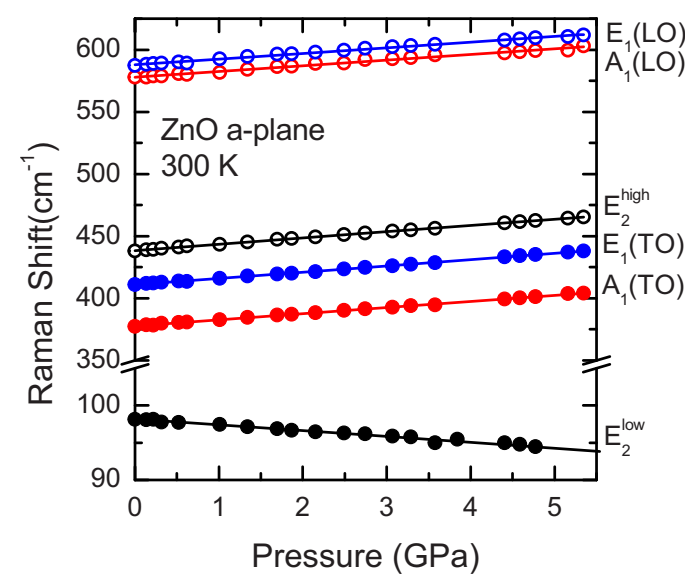

FIG. 2. (Color online) Pressure dependence of the first-order Raman modes. Solid lines are results of least-squares fits to the data points using linear relations.

fits to the data points using a linear relation. Table I shows the results for $\omega_{0}$ and $\partial \omega / \partial P$ together with previous results for comparison. ${ }^{6}$ From the obtained data we computed the mode-Grüneisen parameter as $\gamma_{i}=d\left[\ln \left(\omega_{i}\right)\right] / d[\ln V]$ $\approx\left(B_{0} / \omega_{i}\right) \times \partial \omega_{i} / \partial P$, where $\omega_{i}$ is the mode frequency, $B_{0}$ is the bulk modulus, and $P$ is the pressure. For the isothermal bulk modulus we used the value of $B_{0}=142.6(2) \mathrm{GPa}$, as measured by $\mathrm{x}$-ray diffraction. ${ }^{4}$ The discrepancy in the determination of the Grüneisen parameters with Ref. 6 (see Table I) stems from a different choice of $B_{0}$, and also from the inaccuracy in the determination of the phonon pressure coefficient. Concerning $B_{0}$, Ref. 4 represents the state of the art. The indetermination in the pressure coefficients is substantially reduced in our measurements due to the clear observation of every peak in the Raman spectra measured with the DAC and due to the fitting procedure used to determine the peak positions.

We now turn to the discussion of the LO-TO splitting of the $A_{1}$ and $E_{1}$ phonons. Figure 3 displays the pressure dependence of the LO-TO splitting with similar pressure behavior for both modes. In spite of the scatter of the data, it is clear that the splitting diminishes under pressure in both cases. Concerning the discrepancy between our results and those of Ref. 6, we conclude that the problem lies most probably in the determination of the pressure coefficient of $E_{1}(\mathrm{TO})$. In their case, the $E_{1}(\mathrm{TO})$ phonon was hardly seen at room temperature such that its apparent frequency position was strongly affected by the large asymmetric Fano-like profile of the $E_{2}^{\text {high }}$ mode. ${ }^{15}$

TABLE I. Coefficients from the linear fits to the data points of Fig. 2 using $\omega_{s}=\omega_{0}+(\partial \omega / \partial P) \mathrm{P}$ and corresponding Grüneisen parameters (frequencies in $\mathrm{cm}^{-1}$ and pressures in GPa). For comparison we list results from Ref. 6. Numbers in parenthesis are error bars.

\begin{tabular}{lrrrcc}
\hline \hline Mode & $\omega_{0}$ & $\partial \omega / \partial P$ & $\gamma_{0}$ & $\partial \omega / \partial P^{\text {a }}$ & $\gamma_{0}{ }^{\text {a }}$ \\
\hline$E_{2}^{\text {low }}$ & 98.1 & $-0.78(2)$ & $-1.13(4)$ & -0.93 & -1.6 \\
$E_{2}^{\text {high }}$ & 437.9 & $5.04(3)$ & $1.63(1)$ & 5.16 & 2 \\
$A_{1}(\mathrm{TO})$ & 377.4 & $4.91(5)$ & $1.85(2)$ & 4.72 & 2.1 \\
$E_{1}(\mathrm{TO})$ & 410.9 & $5.03(3)$ & $1.74(1)$ & 4.38 & 1.8 \\
$A_{1}(\mathrm{LO})$ & 577.9 & $4.56(7)$ & $1.12(2)$ & $\ldots$ & $\ldots$ \\
$E_{1}(\mathrm{LO})$ & 587.2 & $4.55(10)$ & $1.11(3)$ & 4.78 & 1.4 \\
\hline \hline
\end{tabular}

${ }^{\mathrm{a}}$ Reference 6 . 


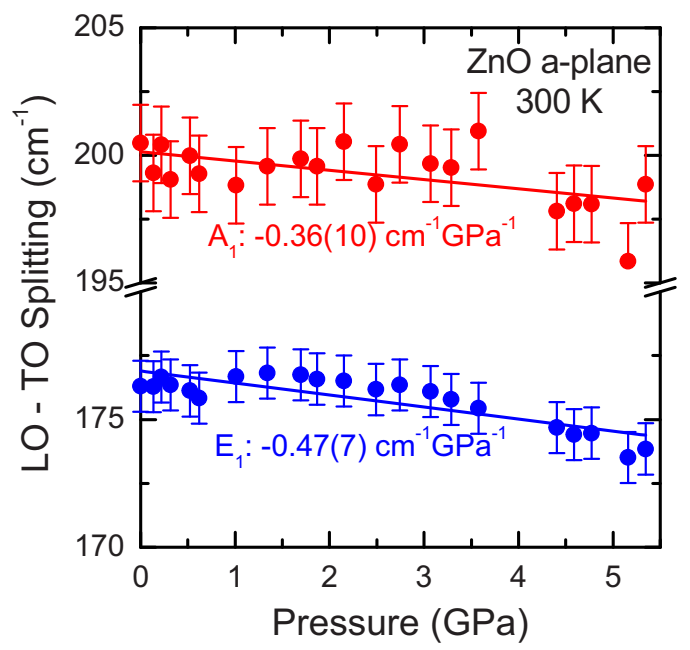

FIG. 3. (Color online) Pressure dependence of the LO-TO splitting for the polar $A_{1}$ and $E_{1}$ phonons. Solid lines are results of least-squares fits to the data points using linear relations.

The Born's transverse effective charge can be calculated according to (in SI units) the following: ${ }^{5}$

$$
\left(e_{T}^{*}\right)^{2}=\varepsilon_{0} \varepsilon_{\infty} V \mu\left(\omega_{\mathrm{LO}}^{2}-\omega_{\mathrm{TO}}^{2}\right),
$$

where $\varepsilon_{0}$ is the vacuum permittivity, $\varepsilon_{\infty}$ is the high frequency dielectric constant, $\mu$ is the reduced mass of the anion-cation pair, $V$ is the volume per pair, and $\omega_{\mathrm{LO}, \text { TO }}$ is the corresponding phonon frequency. For the pressure dependence of the unit-cell volume we used the Murnaghan equation of state ${ }^{16}$ and the bulk modulus from Ref. 4. Unfortunately, for the pressure dependence of $\varepsilon_{\infty}$ there are no high-pressure data available. Nevertheless, we used the pressure dependence of the refractive index at optical frequencies $n_{0}$ measured in the range up to $0.7 \mathrm{GPa}$ (Ref. 17) to obtain $\left(\partial \varepsilon_{\infty} / \partial P\right)$ $=-0.014 \mathrm{GPa}^{-1}$. A close inspection of the Eq. (1) indicates that for $\mathrm{ZnO} e_{T}^{*}$ must decrease with increasing pressure since all involved parameters do as well. The relative contribution of each of these parameters to the pressure dependence of $e_{T}^{*}$ is readily estimated to be about $17 \%, 29 \%$, and $54 \%$ for the mode splitting, the dielectric constant, and the volume, respectively. The results obtained for the transverse effective charge of $\mathrm{ZnO}$ normalized to its zero-pressure value are plotted in Fig. 4 as a function of pressure together with the data for $\mathrm{SiC}, \mathrm{AlN}, \mathrm{GaN}$, and GaAs extracted from Refs. 8, 9, and 11. For completeness the values at ambient pressure are $e_{T}^{*}(0)\left(A_{1}\right)=2.17 \times 10^{-3} e_{0}$ and $e_{T}^{*}(0)\left(E_{1}\right)=2.04 \times 10^{-3} e_{0}$, with $e_{0}$ the elementary electron charge. We point out that there is a clear systematic in the magnitude and sign of the pressure coefficient of the transverse effective charge for the series $\mathrm{SiC}, \mathrm{AlN}, \mathrm{GaN}$, and $\mathrm{ZnO}$ (see Fig. 4). Considering the bond polarity $\alpha_{p}$ as defined by Harrison ${ }^{12}$ we obtain for the previous series $\alpha_{p}=0.26,0.58,0.60$, and 0.78 , respectively. This might indicate that for the heteropolar semiconductor compounds of the form $A^{N} B^{8-N}$ with anion species belonging to the first row of the periodic table the transverse effective charge exhibits the following lattice-constant scaling: for bond polarities lower than $0.6, e_{T}^{*}$ increases with pressure in inverse proportion to the bond polarity. Otherwise, the decrease in $e_{T}^{*}$ is the stronger, the larger the polarity.

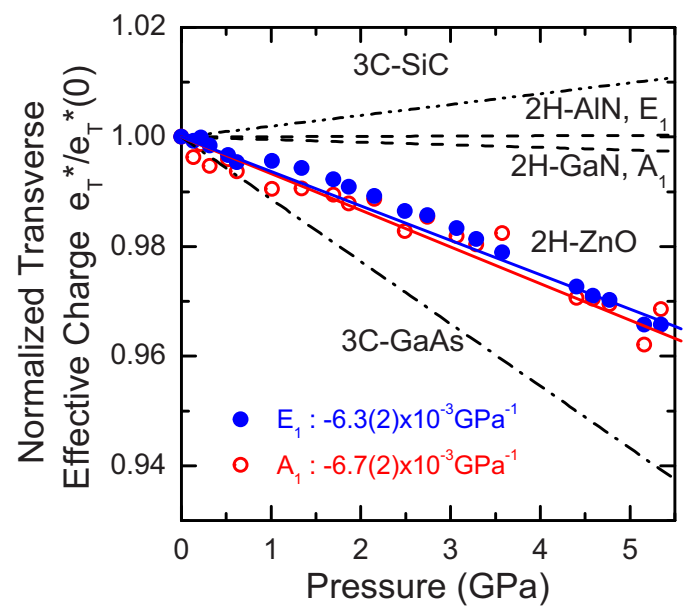

FIG. 4. (Color online) Dependence on pressure of the transverse effective charge normalized to its ambient pressure value. For comparison the dotted lines correspond to the experimental results obtained for $\mathrm{SiC}, \mathrm{AlN}, \mathrm{GaN}$, and GaAs extracted from Ref. 8 .

In conclusion, we have revisited the pressure dependence of the zone center optical phonons of $\mathrm{ZnO}$ using wurtzite a-plane crystals. The LO-TO splitting of the polar modes $E_{1}$ and $A_{1}$ exhibit a moderate decrease with increasing pressure. Consequently, the Born's transverse effective charge also decreases at reduced volumes and its pressure coefficient fits well into a systematic observed for the semiconductor compound series of $\mathrm{SiC}, \mathrm{AlN}, \mathrm{GaN}$, and $\mathrm{ZnO}$ as a function of bond polarity.

A.R.G. is an ICREA Research Professor. This work was supported in part by the Spanish Ministerio de Ciencia e Innovación through Grant No. MAT2009-09480 and Acciones Integradas Hispano-Alemanas 2007, and by DFG within Grant No. SFB787. Measurements were performed at MATGAS 2000 A.I.E.

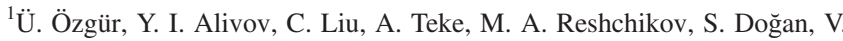
Avrutin, S.-J. Cho, and H. Morkoç, J. Appl. Phys. 98, 041301 (2005).

${ }^{2}$ H. J. Bolink, E. Coronado, D. Repetto, and M. Sessolo, Appl. Phys. Lett. 91, 223501 (2007).

${ }^{3}$ C. Lao, Y. Li, C. P. Wong, and Z. L. Wang, Nano Lett. 7, 1323 (2007).

${ }^{4}$ S. Desgreniers, Phys. Rev. B 58, 14102 (1998).

${ }^{5}$ P. Y. Yu and M. Cardona, Fundamentals of Semiconductors (Springer, Berlin, 1996), p. 293.

${ }^{6}$ F. Decremps, J. Pellicer-Porres, A. M. Saitta, J. C. Chervin, and A. Polian, Phys. Rev. B 65, 092101 (2002).

${ }^{7}$ S. S. Mitra, O. Brafman, W. B. Daniels, and R. K. Crawford, Phys. Rev. 186, 942 (1969).

${ }^{8}$ A. R. Goñi, H. Siegle, K. Syassen, C. Thomsen, and J.-M. Wagner, Phys. Rev. B 64, 035205 (2001).

${ }^{9}$ R. Trommer, H. Müller, M. Cardona, and P. Vogl, Phys. Rev. B 21, 4869 (1980)

${ }^{10}$ B. A. Weinstein and G. J. Piermarini, Phys. Rev. B 12, 1172 (1975).

${ }^{11}$ D. Olego, P. Vogl, and M. Cardona, Phys. Rev. B 25, 3878 (1982).

${ }^{12}$ W. A. Harrison, Electronic Structure and The Properties of Solids (Dover, New York, 1989), p. 175.

${ }^{13}$ H. K. Mao, J. Xu, and P. M. Bell, J. Geophys. Res. 91, 4673 (1986)

${ }^{14}$ R. Cuscó, E. Alarcón-Lladó, L. Artús, J. Ibáñez, J. Jimenez, B.-G. Wang, and M. J. Callahan, Phys. Rev. B 75, 165202 (2007).

${ }^{15}$ J. Serrano, F. J. Manjon, A. H. Romero, F. Widulle, R. Lauck, and M Cardona, Phys. Rev. Lett. 90, 055510 (2003).

${ }^{16}$ F. D. Murnaghan, Proc. Natl. Acad. Sci. U.S.A. 30, 244 (1944).

${ }^{17}$ K. Vedam and T. A. Davis, Phys. Rev. 181, 1196 (1969). 